El maltrato infantil por negligencia: conceptos y visión general sobre su evaluación.

Child abuse by negligence: concepts and overview of evaluation 


\title{
El maltrato infantil por negligencia: conceptos y visión general sobre su evaluación ${ }^{1}$ Child abuse by negligence: concepts and overview of evaluation
}

\author{
Jesús Armando Delgado Meza² \\ Corporación Universitaria de Investigación y Desarrollo -UDI, Bucaramanga, Colombia
}

Artículo recibido en enero de 2016; artículo aceptado en marzo de 2016

Citación del artículo: Delgado, J. (2016). El maltrato infantil por negligencia: conceptos y visión general sobre su evaluación. I+D Revista de Investigaciones, 7(1), 14-23.

\begin{abstract}
Resumen
El presente artículo da cuenta sobre el estado del arte de la producción académica representativa sobre el concepto de maltrato infantil por negligencia y su evaluación, desarrollada en distintos países de América y Europa, hecha por expertos en esta temática. Basado en una metodología cualitativa de análisis documental, que permitió recopilar, sistematizar y comparar teoría sobre el tema desde una perspectiva crítica; se consultaron artículos publicados en revistas y bases de datos aceptadas por la comunidad científica abordándose desde dos ámbitos: uno como productos de investigación y otro como análisis y reflexión, resultado de la profundización sobre el tema por parte de algunos investigadores reconocidos en la literatura. La revisión evidencia avances en la definición legitimada de "conducta negligente" y el fomento de la capacitación a profesionales en técnicas y herramientas para una evaluación más objetiva.
\end{abstract}

Palabras clave: Maltrato infantil, negligencia, definición y valoración

\section{Abstract}

This article reports on the state of the art of most relevant academic research related to child abuse by neglect and its evaluation by experts across different countries of America and Europe. Based on a qualitative methodology of document analysis, the paper exhibit a critique perspective supported on a set of published journal articles and databases accepted by the scientific community. Two main areas of interest were considered, the first one: research products and the other one: a deepening process on the subject of study made by some relevant researchers on the field. This literature review provide evidence about the legitimization of the definition of "negligent conduct " and also support the idea of promoting professional training in techniques and tools related to this field in order to be prepared to more objective assessments.

Keywords: Infant maltreatment, child abuse, negligence, definition and evaluation.

\section{Introducción}

La violencia y el maltrato hacia la población infantil son una preocupación que cada día inquieta más a la sociedad. En los últimos años tanto entidades legales, educativas y de salud se han orientado hacia la protección a niñas, niños y jóvenes, ofreciendo programas de atención y prevención para la población infantil (Urrusono \& Rodríguez, 2009). A pesar de los esfuerzos realizados, sigue siendo una problemática social que se presenta en todos los estatus sociales y en muchas regiones del mundo, incluyendo países desarrollados (Muñoz, 2006; Gilbert et al, 2009).

El maltrato infantil ha sido objeto de investigación de

\footnotetext{
${ }^{1}$ Artículo de revisiónde enfoque cualitativo, resultado del proyecto de investigación terminado denominado: Construcción y validación de un sistema de evaluación de maltrato infantil desencadenado por conductas de negligencia por parte del cuidador, aplicado en niños de 7 a 11 años;perteneciente al área depsicología, sub área de Psicología de la Violencia, desarrollado en el grupo de investigación UDIPSI categoría CColciencias y financiado por la Corporación Universitaria de Investigación y Desarrollo -UDI de la ciudad de Bucaramanga (Colombia). Dirección Calle 9 \# 23 - 55, PBX: 6352525.Fecha de inicio: Febrero de 2012, fecha de terminación: Junio de 2015.

${ }^{2}$ Psicólogo de la Universidad Pontificia Bolivariana. Magíster en Administración de Empresas de la Universidad Viña del Mar y Especialista en Psicología Clínica y de la Salud de la Universidad Autónoma de Bucaramanga. Docente- investigador del grupo: UDIPSI de la Corporación Universitaria de Investigación y Desarrollo-UDIde la ciudad de Bucaramanga (Colombia); Dirección: Calle 9 \# 23 - 55, PBX: 6352525. Correo electrónico institucional:jesus.delgado@udi.edu.co
} 
años atrás, siendo uno de los fenómenos que, junto a la violencia, ha despertado interés desde todas las esferas sociales, políticas, científicas y académicas intentando interpretar su génesis y perpetuidad para llegar a una comprensión con el fin de poder formular propuestas de prevención e intervención (Pineda \& Otero, 2004). La Organización Mundial de la Salud (OMS, 2009) lo considera un problema social creciente que ha llevado a que diferentes países formulen estrategias que en primera instancia eviten su presencia en la familia, para luego llevarlo a niveles sociales, proponiendo formas de intervención que busquen desestimar su continuidad en una sociedad en donde prima la agresión y la violencia.

Las ciencias sociales y humanas, al igual que otras disciplinas, han contribuido a lo largo de los años a hacer un análisis de este fenómeno, estableciendo hoy en día una variedad de maltratos a los que son sometidos los menores de edad, incluyendo los de carácter físico, psicológico, abuso sexual, desatención, negligencia y explotación comercial o de otro tipo que causan o puedan causar un daño a la salud, desarrollo o dignidad del niño, poner en peligro su supervivencia o exponerlo a la muerte.

En una retrospectiva del concepto de maltrato,25 años atrás se definía como un fenómeno secuela de desórdenes psicológicos que acompañaban a los cuidadores y como consecuencia de prácticas de socialización en donde era aceptado el uso de comportamientos agresivos o de violencias y estrategias de poco poder asertivo con los hijos (Gracia, 2002). Como subrayó Rogosch, F.(1995), las experiencias de malos tratos pueden entenderse como la línea extrema de una matriz más general de conductas y orientaciones de los padres y, en ese sentido, el maltrato sería la expresión límite de unas características parentales que son poco efectivas para promover el desarrollo infantil de manera óptima.

Actualmente no se ha desestimado la reflexión que hace tiempo acompaña el considerar los efectos nocivos que produce cualquier expresión de maltrato, ya sea por negligencia o abandono, maltrato físico, abuso sexual y cualquier otro tipo de acción que atente contra la salud mental o psicológica (Mesa \& Moya, 2011), las consecuencias convergen en una serie de daños emocionales severos y duraderos que conllevan incluso a un aumento en problemas de salud (Clark \& Clark, 2001).

Según el diario Vanguardia Liberal, en el informe realizado por la directora del ICBF durante la campaña "por las buenas si aprendo" en Mayo de 2015,refiere que los motivos más comunes de vulneraciones contra los menores corresponden a un $21,2 \%$ por maltrato infantil, $13.5 \%$ por violencia sexual, $5.7 \%$ por abandono y $5 \%$ por consumo de sustancias psicoactivas. El maltrato infantil y la violencia sexual, que corresponden a los de más alto índice, y el tercero, el abandono, son tipos de transgresiones tipificadas hacia los menores que por su manifestación son "fácilmente" evidenciables y que excluyen otro tipo de maltrato como es la negligencia, que por ser tácito y enmascarado son de difícil detección. Este último tipo de maltrato, que corresponde a una de las expresiones más comunes y frecuentes de maltrato infantil, poco es soportado por datos estadísticos que reflejen su estado actual.

Es por ello que pretendiendo conocer aún más sobre las definiciones, características y valoración del maltrato infantil por negligencia se proyecta a través de este texto tener una revisión panorámica que identifique, sintetice y promueva un concepto y evaluación más objetivo sobre este tipo de maltrato.

\section{Método}

Se llevó a cabo un estudio cualitativo, basado en una revisión bibliográfica que permitió una exploración, recopilación y construcción de ideas tratadas desde una perspectiva crítica sobre los conceptos o definiciones a propósito del maltrato infantil por negligencia y estrategias de evaluación utilizados en diferentes países de Europa y América en el que se establecieron como unidades de análisis, libros y artículos científicos desde el año 1972 a 2012. Como unidades de estudio se tomaron como base los títulos de documentos, autores, objetivo general del estudio, país y resultados. Esto permitió hacer una categorización con fines de realizar la revisión sistemática, entre lo que se tuvo en cuenta los conceptos o definiciones sobre maltrato por negligencia, características descritas sobre negligencia y estrategias de evaluación de este tipo de maltrato.

El proceso de revisión inició con la consulta en distintas bases electrónicas de acceso libre que permitió una adecuada recopilación teórica: Redalyc, Scielo, Psicoinfo, Google Scholar, de Marzo a Mayo de 2015. A tal efecto se combinaron las palabras clave de la siguiente manera: maltrato infantil (infant maltreatment) y negligencia (negligence), abuso infantil (child abuse) y negligencia (negligence), maltrato infantil (infant maltreatment) $\mathrm{y}$ evaluación (assesment), y abuso infantil (child abuse) y evaluación (assesment). Posteriormente, se revisaron referencias de varios estudios previos sobre maltrato infantil por negligencia que permitieron el registro de datos en el apartado de este documento denominado "resultados" sobre los distintos conceptos y evaluaciones que sobre el maltrato infantil por negligencia se tienen y se consideran en diferentes países.

El número total de referencias identificadas por los diferentes medios fue de 103.El resultado del proceso de búsqueda permitió seleccionar 70 artículos que cumplían con los criterios de selección de acuerdo a un examen de pertinencia.

Finalmente, para el análisis de los documentos se integró la información obtenida en las diferentes clasificaciones delimitadas en las unidades de análisis y categorías definidas, lo que condujo a la elaboración de un 
informe que permitiese la redacción y organización de la discusión referente al análisis de la literatura.

\section{Resultados}

Dentro de los documentos encontrados se evidencia que un número amplio de ellos exploran aspectos relacionados con las características del maltrato infantil por negligencia, así como los conceptos que se han suscitado alrededor de este tipo de maltrato y sus formas de evaluación en donde juegan un papel importante el momento histórico e interpretativo del fenómeno consultado, la cultura y las normas establecidas para regularla. Con el ánimo de generar una discusión con respecto a las dos categorías de análisis establecidas en esta revisión (concepto de maltrato infantil por negligencia y evaluación), se expondrá a continuación cada una de ellas.

\section{Conceptos de maltrato infantil por negligencia}

La no existencia de una caracterización objetiva de este tipo de maltrato ha dificultado la interpretación e identificación del problema, llevando a que se subjetiven los signos y síntomas y se confundan con algunas otras anomalías. Algunas definiciones son representativamente significativas y expresan la dimensión real de dicho maltrato. Arruabarrena, M. (2011), por ejemplo, ha considerado la negligencia como una situación de desprotección en donde han sido vulneradas las necesidades fisiológicas y físicas básicas como la alimentación, vestido, higiene, protección y vigilancia en circunstancias potencialmente riesgosas o peligrosas, los cuidados médicos y educación, entre otros. A este grupo de necesidades se añaden las emocionales o psíquicas del niño, definida como falta de atención e interés hacia las necesidades de interrelación y afecto, estimulación, orientación y guía del niño, y la no aceptación o rechazo en la proporción de atención psicológica a anomalías emocionales graves como consecuencias del accionar de los padres, incluyendo en algunos casos las acciones de las personas que hacen parte de la unidad de convivencia la cuales no otorgan atención temporal oportuna o permanente.

Por otro lado, Barudy, J. (1998) señaló que la negligencia implica que los responsables del menor asumen de manera incorrecta las funciones de cuidar y proteger, consciente o inconscientemente, voluntaria o involuntariamente. El autor infiere que el efecto es el mismo así sea intencional o no, por ende, debe considerarse como negligencia; sin embargo, la acción judicial o penal sí debe diferenciar ambos aspectos. Los padres son los responsables o culpables por no proporcionar los cuidados necesarios, convirtiéndose en un reto el saber si es razonable o constructivo culpar a un padre por un determinado tipo de desatención (Dubowitz, 2006).

En un contexto más actualizado, las investigaciones refieren que la negligencia corresponde a la incapacidad que tiene el cuidador de otorgar a un menor las condiciones educativas, nutricionales, sanitarias, de desarrollo psicológico, de alojamiento y protección frente a cualquier eventualidad de peligro (Perea, et al, 2007). Según estos autores es importante no confundir negligencia con situación de pobreza, ya que en la primera los cuidadores niegan al niño los recursos de los cuales disponen. Para diferenciarlas, hay que tener presente las condiciones económicas, sociales y culturales de los individuos y los grupos sociales, para lo cual el profesional que realiza la atención al niño debe proceder con cautela, sensibilidad y ahondar en el estudio de los factores del agresor, de la familia, del entorno social y del niño mismo, a fin de identificar la posibilidad de maltrato por negligencia o cualquier otra de sus manifestaciones, evitando realizar juicios a priori sobre este factor.

Esta serie de definiciones sobre maltrato por negligencia evidencian por sí mismas las perspectivas que identifican distintos enfoques teóricos explicativos que han intentado argumentar acerca de la casuística del abandono físico o negligencia infantil. Algunos de esos modelos son los siguientes:

a) El modelo sociológico que centra su etiología en las situaciones de carencia económica en que se produce. Wolock \& Horowitz, (1984) consideran que en el caso de la negligencia se evidencia una ausencia de habilidades por parte de los padres en el cuidado de los niños y un desconocimiento de sus necesidades; así como una falta de motivación en los progenitores que se manifiesta en un rechazo por satisfacer las necesidades de la familia y en la inadecuada supervisión de los niños (Herrenkohl, Herrenkohl \& Egolf, 1983; Moreno, 2006).

b) El modelo cognoscitivo que expone que este tipo de maltrato corresponde a una situación de desprotección como secuela de errores en el pensamiento o distorsiones cognitivas, esquemas maladaptativos, expectativas y percepciones inadecuadas de los cuidadores o progenitores, en relación a los menores a su cargo (Larrance \& Twentyman, 1983; Crittenden, 1993; Milner, 1995yO' Dougherty-Wrigth, Crowford \& Del Castillo, 2009). De esta misma perspectiva surge el modelo del procesamiento de la información, que plantea que los padres experimentan la realidad e interpretan su significado de manera diferente, seleccionando diversas respuestas de distintos repertorios y poniéndolas en funcionamiento bajo condiciones diferentes a la realidad (Milner,1995; Milner, 1993; citado por Hecht \& Hansen, 2001).

c) El modelo psiquiátrico propuesto por Factor, D \& Wolfe, D. (1990), que considera la presencia de psicopatología en los padres, es decir, la presencia de enfermedades mentales o un síndrome o desorden psicológico específico, como lo indican varios estudios que señalan que los padres presentan una baja autoestima, escasa capacidad de empatía (Cantón \& Cortés, 1997), apatía, inmadurez, retraso mental, depresión reactiva y psicótica (Polansky, De Saix \& Charlín, 1972). Todos ellos indicadores de incapacidad para el desempeño del rol parental y, por tanto, del fracaso a la hora de satisfacer las 
necesidades básicas de los hijos.

d)El modelo de afrontamiento del estrés que hace referencia a la forma de evaluar y percibir las situaciones y/o sucesos estresantes por parte de las familias y una falta de implicación conductual y psicológica, eliminación de actividades y búsqueda de apoyo social inadecuado (Hillson \& Kuiper, 1994).

f)El modelo integral del maltrato infantil mencionado por Kempe, R\&Kempe, C (1996)supone distintos niveles ecológicos que están integrados unos dentro de otros, interactuando en periodos de tiempo y en el que se consideran factores compensatorios como: planificación familiar, satisfacción personal, apego materno/paterno al hijo, escasos sucesos vitales estresantes, apoyo social, armonía marital, buena condición financiera, acceso a programas sanitarios adecuados, intervenciones terapéuticas familiares, etc., que actúen siguiendo un modelo de afrontamiento, el cual impide que los factores estresores que aparecen en las familias, desencadenen una respuesta agresiva hacia sus miembros (Morales, Zunzunegui \& Martínez, 1997).

La mayoría de estos modelos explicativos, muestran una visión parcializada que direccionaría a tratarlo desde una perspectiva que desestima concebirlo desde una integralidad, a excepción del modelo etiopatogénico que se ajusta al modelo integral sugerida por distintos autores que no sólo lo han estudiado a profundidad sino que además lo han evaluado. En un análisis histórico sobre la concepción de este tipo de maltrato, se pueden destacar autores que en su cercanía a los modelos anteriormente expuestos, identifican los factores que están relacionados con la negligencia. Un claro ejemplo de lo anterior es lo referido por Polansky, N. (1985) quien identificó la negligencia como un fenómeno invisible, silencioso, insidioso, provocado por una indiferencia generalizada familiar. Este tipo de maltrato, como concluye este autor, atenta en contra de la satisfacción de un conjunto de necesidades de importancia vital para la formación del desarrollo personal del niño. Dicha forma de maltrato no actúa de manera aislada, sino que es parte de un sistema que, además de tolerar e incluso fomentar la negligencia, legitima las relaciones autoritarias y violentas entre los individuos que la conforman, a la par que las reproduce de generación en generación, violando normas legales y derechos reconocidos mundialmente.

En un artículo más reciente de Loredo et al(2010), en su escrito "Maltrato infantil: una acción interdisciplinaria e interinstitucional en México", resalta además la definición de maltrato infantil por negligencia como la incapacidad que tiene un cuidador de asegurar al niño las condiciones de salud física, nutricionales, educativas, de desarrollo psicológico, de alojamiento y protección frente al peligro, aclarando que sólo se puede hablar de negligencia, cuando las personas responsables del niño no les ofrecen los recursos de los que dispone. Además, señala que este tipo de maltrato puede ser difícil de detectar y que suele confundirse con patologías o perturbaciones psicológicas del menor. Sin embargo, existen elementos que permiten discriminarlos ya que los padres que abusan psicológicamente de sus hijos, en ocasiones se muestran despreocupados y culpan a los niños del problema, mientras que los padres de niños psicológicamente perturbados reconocen el problema y solicitan ayuda para resolverlo.

Moreno, J (2008) al referir a Martínez, A\& De Paúl, J (1993) hace una diferenciación comprensible entre maltrato por negligencia y abandono. Estos autores consideran que el maltrato por negligencia es consecuencia de acciones inadecuadas por parte de los progenitores o cuidadores, ante las necesidades físicas, psicológicas, sociales y educativas del menor a su cargo. A su vez lo consideran propio de aquellas familias en donde existen necesidades adicionales primarias, dándose dicha situación de malos tratos con un carácter consciente e inconsciente como producto de la ignorancia, pobreza, etc.

Por su cuenta Rochet, K. (1998), citado por González, J. (2012),igualmente acentúa la necesidad de diferenciar entre abandono y negligencia ya que tiende a confundirse; en primera instancia la negligencia hace alusión al comportamiento del cuidador que por acción u omisión no cubre las necesidades básicas del niño aun estando en condiciones de hacerlo y es inflexible a las demandas exigidas por el menor; comprende aspectos físicos (alimentación, abrigo, protección contra el peligro) y socio- emocionales (afecto, atención, interacción y aceptación de juegos), vigilancia deficiente, descuido, privación de alimentos, incumplimiento de tratamiento médico, impedimento a la educación, actos destructivos y amenazantes que afectan el desarrollo psicológico; llevando esto a un entorno familiar desestructurado; en tanto el abandono es la potencialización aversiva de todo lo anterior que culmina en el desapego físico y espacial y generalmente en la institucionalización.

Finalmente, antela dificultad de diferenciar la negligencia proporcionada por padres que carecen de recursos económicos de aquellos que no, Runyan, D \& Eckenrode, J. (2004) insisten en "no confundir negligencia con pobreza, ya que sólo puede hablarse de negligencia cuando la familia, o quienes son responsables del niño, no le ofrecen los recursos de los que disponen". En esta última definición, la correcta interpretación de las palabras "recursos" y "disponibilidad" son la clave para lograr diferenciar entre estas condiciones. Los recursos corresponden a aquellos elementos que los cuidadores consideran necesarios utilizar con el menor para cumplir con su responsabilidad. Estos recursos incluyen bienes como también muestras afectivas, enriquecimiento cultural y religioso, de crianza, no limitándose a lo estrictamente económico. También, se considera importante destacar en la definición que la disponibilidad encierra la conciencia, la actitud y la oportunidad de los cuidadores en proveer los recursos anteriormente mencionados; es por ello que, es 
negligente aquel cuidador o responsable de un menor que no pone a disposición los recursos y la conciencia de obrar a favor del niño (Glasser, 2011). Es aquí donde precisamente el profesional debe exacerbar la capacidad, actitud y deseo de ahondar y particularizar los casos que sospecha de negligencia, siendo preciso delimitar la existencia o no de los factores propios del menor, del agresor, de la familia y del entorno social que lo predisponen a ser maltratado; así mismo, es una guía de actuación, para evitar una evaluación superficial de cada indicador de negligencia o cualquier otra forma de violencia (Perea et al, 2007).

\section{Evaluación del maltrato por negligencia}

El maltrato infantil es complejo, lo que ha llevado a que sea estudiado y evaluado independientemente de acuerdo a su tipología ya que cada una de las clasificaciones de maltrato tiene distintos antecedentes y consecuentes (Higgins \& McCabe, 2000; citado por Antequera, 2006).

En el caso del maltrato por negligencia, su evaluación está ligada a la profundidad que exista en el conocimiento de su etiología, desarrollo y secuelas, así como de la definición objetiva y operativa sobre el mismo. El concepto de maltrato infantil por negligencia ha presentado algunas dificultades que afectan la consistencia y exactitud en su evaluación (Arruabarrena. 2009). Esta autora considera que en la definición deben tenerse en cuenta aspectos como la etapa del desarrollo en el que se encuentra el niño o la niña, su estado de vulnerabilidad, el considerar las consecuencias que trae el maltrato como elemento para determinar la valoración de la conducta maltratante, los criterios de conducta que limitan el maltrato del no maltrato, las normas específicas que deben reunirse para considerar un buen padre y las características que definan una atención adecuada. Finalmente, otro aspecto que ha sido debatido en la definición del maltrato infantil corresponde a la mayor o menor importancia que se le debe conceder a los valores o normas culturales en función de los cuáles las conductas se consideran socialmente aceptables y adecuadas.

Todo lo anteriormente mencionado ha hecho que la evaluación psicológica del maltrato por negligencia, evidencie dificultades, si se considera que se trata de un fenómeno que presenta una naturaleza multidimensional, determinada por factores como la frecuencia, temporalidad, gravedad, duración, tipo de conductas maltratantes, la edad del menor en el momento de inicio de los episodios y la naturaleza de la relación con el maltratante (Muela, 2008).

Las dificultades conceptuales y su relación con el proceso evaluador han llevado a la necesidad de concretar y operacionalizar el concepto de maltrato para poder llegar a la construcción de instrumentos de evaluación válidos y confiables que partan de definiciones objetivas y consensuadas sobre maltrato, en donde se procura utilizar diversidad de métodos posibles como las entrevistas, la observación, los cuestionarios que incluyan a todos los implicados (niños, padres o cuidadores, agresor) y agentes sociales (informes policiales, asistencia sanitaria, servicios sociales comunitarios, colegios, vecinos) así como abarcar el mayor número de áreas posibles.

Lo anterior ha permitido además que existan diferentes protocolos para cada situación de maltrato, es decir, si es para la detección temprana, notificación o para efectuar intervención terapéutica (González, Fernández \& Secades, 2004) que son utilizados por los profesionales de la salud y las ciencias sociales, e incluso, más delimitados de acuerdo al área de acción, es decir, que están diseñados para trabajadores sociales, psicólogos o médicos, dependiendo del objetivo que tenga el formato de evaluación; siendo los más utilizados los autoinformes para adultos, niños y adolescentes que son diligenciados ya sea de forma escrita $o$ por medio de entrevistas (Amayaet al, 2000). Actualmente los autoinformes varían de acuerdo a la cantidad y el grado de especificidad de sus ítems y a un periodo de reporte que puede comprender varios meses e incluso el ciclo vital completo, consiguiendo información sobre la conducta de maltrato, la frecuencia, la edad de inicio y su gravedad.

Otra técnica que suele ser utilizada es la observación directa, la cual permite describir de manera casi exhaustiva las conductas de falta de protección y que concluye con la calificación y un conjunto de información obtenida sobre el caso. Este método de recolección de información resulta ser complementario a los autoinformes y sobre todo determinante cuando se trata de categorizar el nivel de gravedad del maltrato e ir más allá de descripciones de orden general.

Ahora bien, cuando se trata de instrumentos que complementan la acción de la observación directa basados en categorías como intensidad, frecuencia, duración, tipos de conducta maltratante, la relevancia del maltrato en función de la edady el grado en el que ha sufrido el niño (American Professional Societyonthe Abuse of Children, 1995; Baker, 2009;Barnett et al., 1991; Brassard \& Donovan, 2006; Glaser, 2002; Hart et al., 2002; McGee \& Wolfe, 1991), son diversos los existentes, como por ejemplo: el Graded Care Profile Scale - GCP (Srivastava \& Polnay, 1997), El Eligibility Spectrum (Ontario Association of Children's Aid Societies, 2006) y el Modified Maltreatment Classification System - MMCS (English y the LONGSCAN Investigators, 1997), siendo este último el instrumento más completo y con mayor apoyo empírico (Villodas et al, 2012).

La evaluación del maltrato infantil por negligencia en distintas partes de Iberoamérica, refiere igualmente la utilización de instrumentos basados en protocolos. En España la comunidad de Madrid ha validado un protocolo para el maltrato infantil como parte del programa de atención al menor, el cual está estandarizado y es utilizado principalmente por trabajadores sociales con fines recursivos para complementar la historia clínica. A través de este instrumento se obtienen datos epidemiológicos que facilitan el proceso de intervención del niño maltratado (Olaya, Tarragona, De la Osa \& Ezpeleta, 2008). 
Para hacer alusión a la evaluación de maltrato por negligencia en otros países, se destaca el protocolo creado en el Hospital de Niños Roberto del Rio de Chile, el cual consiste en una guía clínica del maltrato utilizado por las instituciones públicas y privadas judiciales y de apoyo social, con la finalidad de mejorar la coordinación del trabajo de equipo interdisciplinario en relación al maltrato infantil. Esta guía cuenta con un flujograma que permite seguir un proceso en la toma de decisiones para el manejo del niño agredido, el cual incluye el abordaje clínico en unidad de urgencias, el apoyo psicoterapéutico para el manejo de las consecuencias de maltrato y el manejo en el colegio cuando existe sospecha de otro tipo de maltrato (Moya, Olivari \& Ulloa, 2003).

Finalmente, en Colombia se utiliza el protocolo de atención a la niñez maltratada formulado por la Organización Panamericana de la Salud, el cual consta de una guía informativa con un marco conceptual de maltrato infantil, sus indicadores y una estrategia metodológica para instruir a todos los profesionales tanto de la salud como administrativos en el procedimiento a seguir para la evaluación de cualquier tipo de maltrato (Organización panamericana de la salud, 2006). Esta guía se empezó a utilizar a partir de la Resolución 412 del 2000 del Ministerio de Salud de Colombia y la dirección general de promoción y prevención, estableciendo los criterios técnico-científicos de intervención a través de un protocolo de maltrato infantil necesario para el fomento del buen trato, la detección e intervención de los factores de riesgo, la detección temprana de maltrato y abuso sexual, la atención y el seguimiento de los niños maltratados/abusados, a través de un abordaje integral, interdisciplinario e intersectorial.

\section{Discusión}

La revisión de estudios y trabajos realizados sobre el tema permite dar a conocer más coincidencias que diferencias en las interpretaciones de profesionales e investigadores en la compleja definición, identificación y evaluación de este tipo de maltrato, llegando finalmente a una cercana objetivación de las conductas hacia los hijos que son consideradas legítimas (Schmidt \& Benbenishty, 2011), gracias a que en las últimas décadas se han producido destacados avances para llegar a cumplir con este fin (Arruabarrena, 2011).

La situación descrita en éste documento permite evidenciar una evolución en la definición del maltrato por negligencia que se tenía en el siglo XX, concluyendo que se trata de una incapacidad que tienen los padres o cuidadores de procurar al niño las condiciones físicas-sanitarias, nutricionales, de alojamiento y protección frente a cualquier peligro, de desarrollo psicológico, social y educativas, que debería ser capaz de proporcionar, destacando claramente que sólo puede hablarse de negligencia, cuando la familia o quienes son responsables del niño, no le ofrecen los recursos de los que disponen (Runyan et al (2002).

Finalmente, ante la diversidad de estratégicas utilizadas para la detección de casos de maltrato por negligencia, se hace importante insistir no solamente en la construcción de sistemas objetivos alternativos de evaluación que permitan a los profesionales de la salud, educadores, líderes comunitarios, entre otros, realizar el diagnóstico e intervención de este tipo de maltrato, sino también llevar a cabo una adecuada orientación a la superación de las barreras que no permiten la objetividad en su valoración, recurriendo continuamente a la capacitación en técnicas y herramientas para la recolección de información de manera rigurosa y neutral (Arruabarrena, De Paul, Indias \& Ullate, 2013). De esta manera habrá mayor capacidad de ayuda a los menores víctimas de maltrato, contribuyendo de esta forma a la mejora de la calidad integral del sistema de protección a la infancia y adolescencia (Rodrigo, Maíquez, Martín, \& Byrne, 2008; Arruabarrena \& De Paul,2011).

\section{Referencias}

Amaya-Jackson, L; Socolar, R; Hunter, W; Runyan, D \& Colindres, R. (2000). Directly questioning children and adolescents about maltreatment.A review of survey measures used. Journal of Interpersonal Violence, 15, pp. 725-759.

American professional society on the abuse of children (1995). Psychosocial evaluation of suspected psychological maltreatment in children and adolescents. Practiceguidelines. Chicago: APSAC.

Antequera, R. (2006). Evaluación psicológica del maltrato en la infancia. Cuaderno de medicina forense, 12(43-44),pp.129-148.

Arruabarrena, M. (2009). Procedimiento y criterios para la evaluación y la intervención con familias y menores en el ámbito de la protección infantil. Papeles del Psicólogo, 30, pp.13-23.

Arruabarrena, M. (2011). Maltrato Psicológico a los Niños, Niñas y Adolescentes en la Familia: Definición y Valoración de su Gravedad.vol. 20, núm. 1, 2011, pp. 25-44 Colegio Oficial de Psicólogos de Madrid Madrid, España. Recuperado el 4 d e $\quad M$ arzo de 2015 de:http://www.copmadrid.org/webcopm/publicaciones/so cial/in2011v20n1a3.pdf

Arruabarrena, M \& De Paul, J. (2011). Valoración de la gravedad de las situaciones de desprotección infantil por los profesionales de los servicios de protección. Psicothema, 23, 642-647.

Arruabarrena, I; De Paúl, J; Indias, S \& Ullate, M (2013). Psychologist and child psychologicas maltreatment severity assessment.Psicothema 25(4), pp. 482-7. doi: 10.7334/psicothema2013.146.

Baker, A. (2009). Adult recall of childhood psychological maltreatment: Definitional strategies and 
challenges. Children and Youth Services Review, 31, pp. 703-714.

Barudy, J. (1998). El dolor invisible de la infancia. Una lectura ecosistémica del maltrato infantil.Barcelona: Paidós.

Barnett, D; Manly, J. \&Cicchetti, D. (1991). Continuing toward an operational definition of psychological maltreatment. Development and Psychopathology, 3, pp. 19-30.

Brassard, M.\& Donovan, K. (2006).Defining psychological maltreatment. En M. M. Feerick, J. F. Knutson, P. K. Trickett y S. M. Flanzer (Eds.), Child abuse and neglect. Definitions, classifications, and a framework for research (pp. 151-197). Baltimore: Paul H. Brookes Publishing Co.

Cantón, J\&Cortés, M. (1997). Malos tratos y abuso sexual infantil. Madrid: Siglo XXI.

Clark, R \& Clark, J. (2001), The Encyclopedia of Child abuse, $2^{\mathrm{a}}$ edition, Facts on file, Inc, Nueva York.

Crittenden, P.(1993). An information processing perspective on the behavior of neglectful parents.Criminal Justice and Behavior, 20, pp. 27-49.

Dubowitz, H. (2006). Defining child neglect. En M.M. Feerick,J; H; Knutson, P.K. Trickectt y S.M Flanzer (eds), Child abuse and neglect.Definitions, classifications and a framework for research (pp. 107 - 127).Baltimore, MD: Paul H. Brookes Publishing Co.

English,D\& the LONGSCAN Investigators (1997).Modified Maltreatment Classification System (MMCS). Recuperado 19 de Marzo de 2015de: http://www.iprc.unc.edu/longscan/pages/mmcs/LONGSC AN\%20MMCS\%20Coding.pdf

Factor, D\& Wolfe, D. (1990).Parental psychopathology and high - risk children. En R.T. Ammerman \& M. Hersen (Eds), Children at risk. An evaluation of factors contribuling to child abuse an neglect. Nueva York: Plenum Press.

Glaser, D. (2002). Emotional abuse and neglect (psychological maltreatment): A conceptual framework. Child Abuse \& Neglect, 26, pp. 697-714

Glasser, D. (2011) How to deal with emotional abuse and neglect further development of a conceptual framework (FRAMEA).Child abuse y neglect, 35, pp. 866 -875 .

Gilbert, R; Widom, C; Browne, K; Fergusson, D; Webb, E \& Janson, S. (2009). Burden and consequences of child maltreatment in high - income countries. The lancet 373 , pp. $68-81$.

González, J; Fernández, J \& Secades, H. (2004). Guía para la detección e intervención temprana con menores en riesgo. Editorial Colegio Oficial de Psicólogos del Principado de Asturias. Recuperado de: http://www.pnsd.msssi.gob.es/Categoria2/publica/pdf/Me noresRiesgo.pdf

González, J. (2012). Factores psicosociales del macrosistema asociados al abandono infantil en un grupo de adolescentes de 12 a 18 años institucionalizados en un centro de protección de la ciudad de Bogotá. Recuperado el

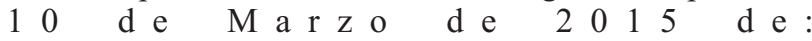
http://bibliotecadigital.usbcali.edu.co/jspui/bitstream/108 19/702/1/Factores_psicosociales_macrosistema_Gonzale z_2008.pdf

Gracia, E. (2002). El maltrato infantil en el contexto de la conducta parental:

Percepciones de padres e hijos. Recuperado el 26 de $\begin{array}{llllll}\mathrm{M} \mathrm{a} \mathrm{r} \mathrm{z} \mathrm{O} & \mathrm{d} \text { e } & 0 & 15 \mathrm{~d} \mathrm{e} \text { : }\end{array}$ http://www.psicothema.com/pdf/720.pdf

Hart, S.; Brassard, M; Binggeli, N \& Davidson, H. A. (2002). Psychological maltreatment. En J. E. B. Myers, L. Berliner, J. Briere, C. T. Hendrix, C. Jenny y T. A. Reid (Eds.), The APSAC handbook on child maltreatment (second edition) (pp. 79-104). Thousand Oaks: Sage Publications.

Hecht, D. \& Hansen, D. (2001). The environment of child maltreatment: Contextual factors and the development of psychopathology. Psychology, Department of Faculty Publications, Department of Psychology.Recuperadoel 2 de Abril de 2015 de: http://digitalcommons.unl.edu/cgi/viewcontent.cgi?article $=1025 \&$ amp; context $=$ psychfacpub

Herrenkohl, R, Herrenkohl, E \& Egolf, B. (1983). Circunstances surrounding the ocurrence of child maltreatment.Journal of Consulting and Clinical Psychology, 51(3), oo.424-431.

Higgins, D.McCabe, M. (2000).Multi type maltreatment and the long term adjustment of adults. Child Abuse Review, 9,pp. 6-18.

Hillson, J \& Kuiper, N. (1994). A Stress and coping model of child maltreatment. Clinical Psychology Reviex, 14,pp. 261-285.

Kempe, R. \& Kempe, C. (1996) Niños maltratados. Madrid: Ediciones Morata.

Larrance, D. \& Twentyman, C. (1983). Maternal attributions and child abuse. Journal of Abnormal Psychology, 92(4), pp. 449-457. 
Loredo, A; Trejo, J; García, C; Portillo, A; Capistrán, A; Carballo, R. \& Martín, V. (2010). Maltrato infantil: Una acción interdisciplinaria e interinstitucional en México. Comisión Nacional para el Estudio y la Atención Integral al Niño Maltratado. Primera parte. Salud mental, 33(3), pp. 281-290.

Martínez, A. \& De Paúl, J. (1993). Maltrato y abandono en la infancia. Barcelona: Martínez Roca.

McGee, R. \& Wolfe, D. (1991). Psychological maltreatment: Toward an operacional definition. Development and Psychopathology, 3, pp. 3-18.

Mesa, P \&Moya, L. (2011). Neurobiología del maltrato infantil: el ciclo de la violencia. Recuperadoel 8 de Abril de 20015

d e : http:/www.neurologia.com/pdf/Web/5208/bf080489.pdf

Milner, J. (1995). La aplicación de la teoría del procesamiento de la información social al problema del maltrato físico a niños. Infancia y Aprendizaje, 71, pp. $125-$ 134.

Morales J, Zunzunegui, V.\& Martínez, V. (1997). Modelos conceptuales del maltrato infantil: una aproximación biopsicosocial. GacSanit11,pp. 231-41.

Moreno, J. (2006). Revisión de los principales modelos teóricos explicativos del maltrato infantil. Enseñanza $e$ Investigación en Psicología, vol. 11, núm. 2, juliodiciembre, 2006, pp. 271-292, México.

Moreno, J. (2008). Estudio sobre las consecuencias del maltrato infantil en el desarrollo del lenguaje. Cuadernos de Psicopedagogia, 7(12), pp. $04-05$

Moya, H., Olivarí. F. \& Ulloa., F. (2003). Maltrato infantil: Guía Clínica Hospital de Niños Roberto del Río. Recuperado el 27 de Mayo de 2015 de http:// w w w. derechosdela infancia.c $1 / \mathrm{docs} /$ imgs/imgs_doc/72.pdf

Muela, A. (2008). Hacia un sistema de clasificación nosológico del maltrato infantil. Anales de Psicología, 24, pp. 77-87.

Muñoz, D. (2006). El maltrato infantil: un problema de salud pública. Cali, Colombia. Recuperado el 27 de Marzo de 2015 de:

Observatorio de infancia: grupo de trabajo sobre el maltrato infantil (2006).

Maltrato infantil: detección, notificación y registro de casos. Ministerio de Trabajo y Asuntos Sociales Edita: Subdirección General de Información Administrativa y Publicaciones Agustín de Bethencourt, 11.28003-Madrid. Recuperado el 5 de Mayo de 2015 de: http://www.observatoriodelainfancia.msssi.gob.es/docum entos/HojasDeteccion.pdf
O' Dougherty-Wrigth, M; Crowford, E \& Del Castillo, D (2009). Childhood emotional maltreatment and later psychological distress among college students: The mediating role of maladaptive schemas.Child Abuse \& Neglect, 33, pp. 59-68.

Olaya, B; Tarragona, M; De la Osa, N., \& Ezpeleta, L. (2008). Protocolo de evaluación de niños y adolescentes víctimas de la violencia doméstica.Papeles del Psicólogo, enero-abril, año/vol. 29, número 001 Consejo General de Colegios Oficiales de Psicólogos Madrid, España. Papeles del Psicólogo, 29(1), pp. 123-135.

Ontario association of children's aid societies.(2006). Eligibility Spectrum. Recuperado de: http://www.oacas.org/pubs/oacas/eligibility/index.htm

Organización panamericana de la salud, Colombia (2006). Protocolo de atención a la niñez maltratada. Documento guía, Cali: Red Colombiana de Municipios Saludables.

Organización mundial de la salud (2009). Prevención del maltrato infantil: qué hacer, y cómo obtener evidencias. III.Organización Mundial de la Salud. IV.Sociedad Internacional para la Prevención del Maltrato y el Abandono de los Niños. ISBN 9789243594361. Recuperadoel 13 de Marzo de 2015 de: http://whqlibdoc.who.int/publications/2009/97892435943 61_spa.pdf

Organización mundial de la salud (2014).Nota descriptiva N. ${ }^{\circ} 150$.Recuperadoel 12 de Marzo de 2015 de: http:/www.who.int/mediacentre/factsheets/fs 150/es/

Perea, A; Loredo, A; Lopez, G; Jordan, N\& Trejo, J. (2007). Negligencia o pobreza: El sobrediagnóstico del maltrato al menor. Acta Pediátrica Mex 2007;28(5), pp. 193 - 7. Recuperado el 2 de Abril de 2015de:http://www.medigraphic.com/pdfs/actpedmex/ap m-2007/apm075e.pdf

Pineda, J \& Otero, L. (2004), Género, violencia intrafamiliar e intervención pública en Colombia. Revista de estudios sociales, No, 17, Febrero de 2004, pp. 19-31.

Polansky, N. (1985). Determinant of loneliness among neglectful and other low-income mother.Journal Social Service Reserch, 8, pp. 1-15.

Polansky, N; De Saix, C. \& Sharlin, S. (1972).Child neglect.Understanding and reaching the parent. Washington, D.C.: Child Welfare League of America.

Rochet, K. (1998).Infancia y maltrato.Buenos Aires: Lumen.

Rodrigo, M; Maíquez, M; Martín, J \& Byrne, S. (2008). Preservación familiar. Un enfoque positivo para la 
intervención con familias. Madrid: Piramide.

Rogosch, F; Cicchetti, D; Shields, A. \&Toth, S. (1995). Parenting dys-function in child maltreatment. En M.H. Bornstein (Ed.), Handbook of parenting. Mahwah: Laurence Erlbaum.

Runyan, D; Wattam, C.; Ikeda, R.;Hassan, F \& Ramiro, L (2002).Child Abuse and Neglect by Parents and Other Caregivers (From World Report on Violence and Health, pp. 57-86, 2002, Etienne G. Krug, Linda L. Dahlberg, et al., eds. -- See NCJ-197425).

Runyan D \& Eckenrode J. (2004). Perspectivas internacionales sobre la epidemiología de la negligencia grave y la violencia en la infancia. Anales Nestlé 2004;62, pp. 1-14.

Schmidt, H. \& Benbenishty, R. (2011). Public attitudes toward child maltreatment in Israel: Implications for policy. Children and Youth Services Review, 33, pp. 1181-1188.

Srivastava, O. \& Polnay, S. (1997). Field trial of graded care profile (GCP) scale: A new measure of care. Archives of Disease in Childhood, 76, pp. 337-340.
Urrusono, F \& Rodríguez, R. (2009). Victimización infantil, un problema de salud en el sistema social capitalista Revista Cubana de Salud Pública, vol. 35, núm. 3, julio-septiembre,pp. 1-5 Sociedad Cubana de Administración de Salud La Habana, Cuba.

Vanguardia.com - Galvis Ramirez y Cía, S.A (19 de Mayo de 2015). ICBF ha atendido 1.391 casos de maltrato infantil durante 2015.Recuperado el 22 de Mayo de 2016de: http://www.vanguardia.com/colombia/312097icbf-ha-atendido-1391-casos-de-maltrato-infantildurante-2015.

Villodas, M; Litrownik, A; Thompson, R.; Roesch, S; English, D; Dubowitz, H \&Runyan, D.(2012). Changes in Youth's Experiences of Child Maltreatment Across Developmental Periods in the LONGSCAN consortium. Psychology of Violence, 2(4), pp. 325-338. doi:10.1037/a0029829.

Wolock, I \& Horowitz, B. (1984). Child maltreatment as a social problem: the neglect of neglect. American Journal of Orthopsyxhiatry, 54(4), pp. 530-543. 